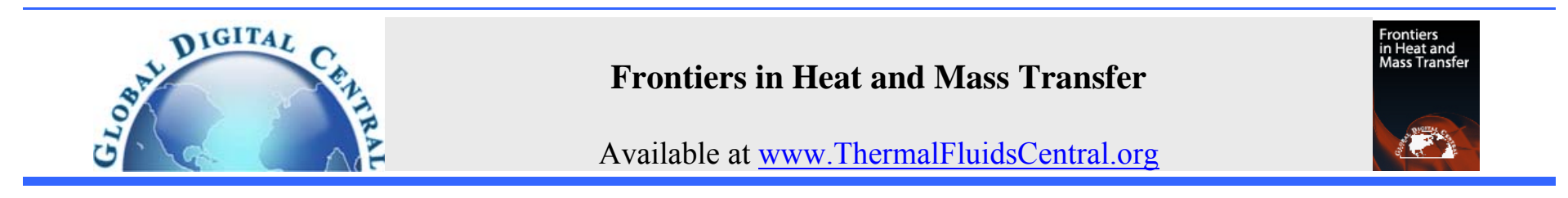

\title{
MHD CONVECTIVE BOUNDARY LAYER FLOW TOWARDS A VERTICAL SURFACE IN A POROUS MEDIUM WITH RADIATION, CHEMICAL REACTION AND INTERNAL HEAT GENERATION
}

\author{
Emmanuel Maurice Arthur*, Timothy Ayando, Yakubu Ibrahim Seini \\ Faculty of Mathematical Sciences, University for Development Studies, P.O. Box 1350, Tamale, +233, Ghana
}

\begin{abstract}
The combined effects of chemical reaction and viscous dissipation on hydromagnetic mixed convective flow towards a vertical plate embedded in a highly porous medium with radiation and internal heat generation has been examined. The governing boundary layer equations have been transformed to a two-point boundary value problem using a local similarity approach and solved numerically using the Newton Raphson shooting method alongside the Fourth-order Runge - Kutta algorithm. The effects of various embedded parameters on fluid velocity, temperature and concentration have been presented graphically whilst the skin friction coefficient and the rates of heat and mass transfers have been tabulated for varying controlling parameters. The results indicate that the magnetic parameter, the local Grashof numbers and all the controlling parameters identified directly influence the flow around the boundary and hence can be controlled to achieve desired product characteristics.

Keywords: Viscous Dissipation, Similarity Variable, Permeability, Brinkman Number.
\end{abstract}

\section{INTRODUCTION}

The subject of coupled heat and mass transfer due to hydromagnetic mixed convection flow past a surface embedded in a highly porous medium have gained considerable attention in the recent past due to their diverse applications in engineering systems. MHD applications are common in the cooling of electronic equipment, nuclear reactors; food processing; polymer production; electromagnetic pumps, valves for transportation, batching, stirring metals- which affect the convective heat and mass transfer in crystallization process in metallurgy- and in liquid metal cooling systems for nuclear technology. Furthermore, MHD plays an important role in building of power engineering devices, metallurgical and electrical technologies; MHD relays, communication switches, and units of automatics with a liquid working body.

The analysis of hydromagnetic flows in porous media has been a subject of several recent papers (Kumari et al. (2001); Chamkha (2001); Seddeek (2002); Arthur and Seini (2014a), Christian et al. (2014), Seini and Makinde (2014)). Most studies on hydromagnetic convection in porous media consider the condition of external flows. It is worth mentioning that, stagnation points also occurs at the surface of objects in the flow field, where the fluid attains a zero velocity. Stuart (1959) examined viscous flow near stagnation point when the external flow has uniform vorticity. Tamada (1979) studied two-dimensional stagnation point flow impinging obliquely on a plane wall. Ali et al. (2011) then analyzed the effect of Hall current on MHD mixed convection boundary layer low over a stretched vertical flat plate while Hayat et al. (2004) examined some steady MHD flows of the second order grade fluid. Ishak et al. (2006) studied mixed convection boundary layers in the stagnationpoint flow toward a stretching surface and later Ishak et al. (2008) examined mixed convection stagnation point flow of a micropolar fluid towards a stretching sheet. Singh et al. (2010) studied the effects of volumetric heat generation/absorption on mixed convection stagnation point flow on an isothermal vertical plate in a porous media.

Radiative flow of MHD fluid occurs in electrical power generation, astrophysical flows, solar power technology and nuclear reactors. Also radiative heat and mass transfer arises in many geothermal and geophysical processes. Thermal radiation is a characteristic of any flow system at temperatures above the absolute zero and can strongly interact with convection in many situations of engineering interest. The effects of radiation on flow kinematics in the presence or absence of magnetic field under various conditions have been studied by a number of researchers. Chamkha et al. (2002) analyzed the effects of radiative heat transfer on flow and thermal field for horizontal and inclined plates. The effect of thermal radiation on the heat and mass transfer flow of variable viscosity fluid past a vertical porous plate permeated by a transverse magnetic field was reported by Makinde and Ogulu (2008). The hydromagnetic flow in the presence of radiation has been investigated by Bestman and Adiepong (1988), Naroua et al. (1998) and Ouaf (2005) when the induced magnetic field is negligible. Raptis et al. (2003) investigated the effects of radiation in an optically thin gray gas past a vertical infinite plate in the presence of a magnetic field, when the magnetic Reynolds number was negligible and the flow was steady. The effect of thermal radiation in the linearized Rosseland approximation on the heat transfer characteristics of various boundary layer flows was reported by Magyari and Pantokratoras (2003). More recently, Arthur et al. (2014) reported on chemically reacting hydromagnetic flow over a flat surface in the presence of radiation with viscous dissipation and convective boundary conditions.

Viscous dissipation plays an important role in natural convection, geological processes, polymer processing and in strong gravitational field processes on large scales and has been reported extensively in the literature. It plays a practical role in oil products transportation through ducts. Viscous dissipation changes the temperature distribution by playing a role like an energy source, which leads to affected heat transfer 
rate and hence needs to be considered in heat transfer problems. Some researchers have examined the effects of viscous dissipation on hydrodynamics with or without magnetic field, notably Pantokratoras (2004), Gangadhar (2012), Ibrahim and Bhashar (2013), Singh (2012), Kazi et al. (2013), Arthur and Seini (2014b), and Imoro et al. (2014).

The objective of this study is to extend the work of Makinde (2011) to include viscous dissipation and chemical reaction. The governing boundary layer equations have been transformed to a two-point boundary value problem using a local similarity approach and solved numerically. The effects of various embedded parameters on fluid velocity, temperature and concentration have been shown graphically. It is hoped that the results obtained will not only provide useful information for application and future research work, but also serve as a complement to the previous study.

\section{MATHEMATICAL MODEL}

We consider a steady, laminar, stagnation point flow of viscous incompressible hydromagnetic fluid through a porous medium along a vertical isothermal plate in the presence of volumetric rate of heat generation. It is assumed that the fluid property variations due to temperature and chemical species concentration are limited to fluid density. In addition, there is no applied electric field and all of the Hall effects and Joule heating are neglected. Since the magnetic Reynolds number is very small for most fluids used in industrial applications, we assume that the induced magnetic field is negligible. The $x$-axis is taken along the plate and the $y$-axis is normal to the plate and the flow is confined in half plane $y>0$ as shown in Fig. 1 .

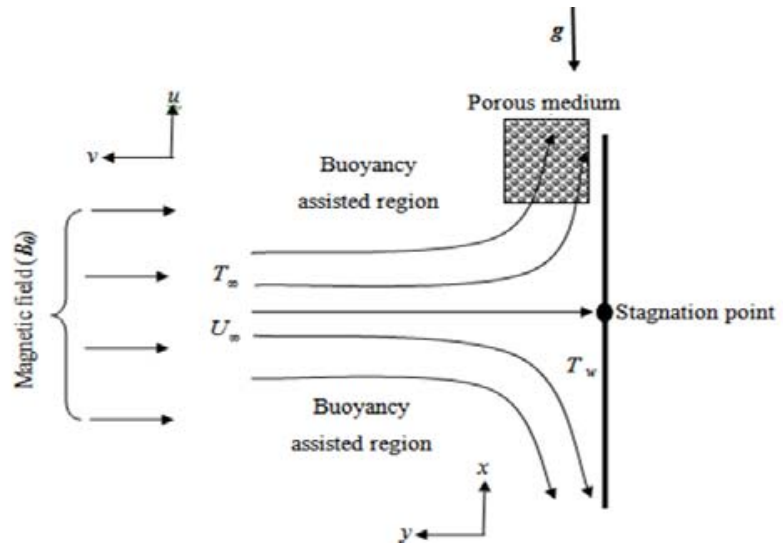

Fig. 1 Schematic Diagram of the Problem

The potential flow arrives from the $y$-axis and impinges on the plate, which divides at the stagnation point into two streams and the viscous flow adheres to the plate. The velocity in the potential flow is given by $U_{\infty}=b x$, where $b$ is a positive constant. Following the linear Darcy term representing distributed body force due to porous media is retained while the non-linear Forchheimer term is neglected with $u, v, T$ and $C$ being the fluid $x$-component of velocity, $y$ component of velocity, temperature and concentration respectively, the governing equations of the continuity, momentum, energy, and species concentration are given by:

$$
\begin{gathered}
\frac{\partial u}{\partial x}+\frac{\partial v}{\partial y}=0 \\
u \frac{\partial u}{\partial x}+v \frac{\partial u}{\partial y}=v \frac{\partial^{2} u}{\partial y^{2}}+g \beta_{T}\left(T-T_{\infty}\right)+g \beta_{C}\left(C-C_{\infty}\right)- \\
\left(\frac{\sigma B_{\circ}^{2}}{\rho}+\frac{v}{\widetilde{K}}\right)\left(u-U_{\infty}\right)+U_{\infty} \frac{\partial U_{\infty}}{\partial x},
\end{gathered}
$$

$$
\begin{gathered}
u \frac{\partial T}{\partial x}+v \frac{\partial T}{\partial y}=\alpha \frac{\partial^{2} T}{\partial y^{2}}+\frac{v}{c_{p}}\left(\frac{\partial u}{\partial y}\right)^{2}-\frac{\alpha}{\kappa} \frac{\partial q_{r}}{\partial y}+Q\left(C-C_{\infty}\right), \\
u \frac{\partial C}{\partial x}+v \frac{\partial C}{\partial y}=D \frac{\partial^{2} C}{\partial y^{2}}-\gamma\left(C-C_{\infty}\right)^{n} .
\end{gathered}
$$

Subject to the following boundary conditions:

$u(x, 0)=0, v(x, 0)=0, T(x, 0)=T_{w}, C(x, 0)=C_{w}$,

$u(x, \infty)=U_{\infty}=b x, T(x, \infty)=T_{\infty}, C(x, \infty)=C_{\infty}$.

where $v$ is the kinematic viscosity, $T_{\infty}$ is the free stream temperature, $C_{\infty}$ is the free stream concentration, $U_{\infty}$ is the free stream velocity, $\alpha$ is the thermal diffusivity and $D$ is the mass diffusivity, $\beta_{T}$ is the thermal expansion coefficient, $\beta_{C}$ is the solutal expansion coefficient, $\rho$ is the fluid density, $g$ is gravitational acceleration, $\sigma$ is the electrical conductivity, $c_{p}$ is the specific heat capacity at constant pressure, $\kappa$ is the thermal conductivity, $\gamma$ is the rate of chemical reaction, $\check{K}$ is the permeability of the porous medium, $n$ is the order of chemical reaction, $Q$ is the volumetric rate of heat generation, $B_{0}$ is the magnetic field of constant strength, $T_{w}$ is the temperature of the plate, and $C_{w}$ is the species concentration at the plate surface.

The velocity components can be expressed in terms of the stream function $\psi(x, y)$ such that

$$
u=\frac{\partial \psi}{\partial y}, v=-\frac{\partial \psi}{\partial x}
$$

We seek for the stream function such that

$$
\psi(x, y)=x \sqrt{v b} f(\eta)
$$

It may be verified that the continuity equation in Eq. 1 is identically satisfied. Using the Rosseland approximation for radiation, Ibrahim and Makinde (2011) simplified the heat flux as:

$$
q_{r}=-\frac{4 \sigma^{*}}{3 K^{\prime}} \frac{\partial T^{4}}{\partial y}
$$

where $K^{\prime}$ and $\sigma^{*}$ are the Stefan-Boltzmann constant and the mean absorption coefficient respectively. We assume that the temperature differences within the flow such as the term $T^{4}$ may be expressed as a linear function of temperature. Hence, expanding $T^{4}$ in a Taylor series about $T_{\infty}$ and neglecting higher order terms, we get;

$$
T^{4} \cong 4 T_{\infty}^{3} T-3 T_{\infty}^{4}
$$

Also, the following non-dimensional variables can be introduced:

$$
\eta=y \sqrt{\frac{b}{v}}, \theta(\eta)=\frac{T-T_{\infty}}{T_{w}-T_{\infty}}, \phi(\eta)=\frac{C-C_{\infty}}{C_{w}-T_{\infty}} .
$$

Considering Eq.6, Eq.8 and Eq.10, Eq.2 - Eq.4 can be transformed into the following ordinary nonlinear system of differential equations:

$$
\begin{gathered}
f^{\prime \prime \prime}+f f^{\prime \prime}-f^{\prime 2}+G_{T} \theta+G_{C} \phi-(M+K)\left(f^{\prime}-1\right)=-1, \\
\left(1+\frac{4}{3} R a\right) \theta^{\prime \prime}+\operatorname{Pr} f \theta^{\prime}+B r f^{\prime \prime 2}+S \phi=0, \\
\phi^{\prime \prime}+S c f \phi^{\prime}-S c \beta \phi^{n}=0 .
\end{gathered}
$$

with the transformed boundary conditions as;

$f^{\prime}(0)=0, f(0)=0, \theta(0)=1, \phi(0)=1$,

$f^{\prime}(\infty)=1, \theta(\infty)=0, \phi(\infty)=0$.

where the prime symbol denotes differentiation with respect to $\eta$ and $G_{T}=\frac{g \beta_{T}\left(T_{w}-T_{\infty}\right) x^{3}}{v^{2}}$ is the local thermal Grashof number,

$G_{C}=\frac{g \beta_{C}\left(C_{w}-C_{\infty}\right) x^{3}}{v^{2}}$ is the local solutal Grashof number,

$B r=\frac{\mu U_{0}^{2}}{\kappa\left(T_{w}-T_{\infty}\right)}$ is the Brinkmann number, 
$\operatorname{Pr}=\frac{v}{\alpha}$ is the Prandtl number,

$S c=\frac{v}{D}$ is the Schmidt number,

$\beta=\gamma\left(C_{w}-C_{\infty}\right)^{n-1} / b$ is the chemical reaction parameter,

$K=\frac{v}{b \widetilde{K}}$ is the permeability parameter,

$S=\frac{Q\left(C_{w}-C_{\infty}\right) v}{b \alpha\left(T_{w}-T_{\infty}\right)}$ is the heat generation parameter,

$M=\frac{\sigma B_{0}^{2}}{b \rho}$ is the magnetic field parameter, and

$R a=\frac{4 \sigma^{*} T_{\infty}^{3}}{\kappa K^{\prime}}$ is the thermal radiation parameter.

\section{NUMERICAL PROCEDURE}

The numerical technique used to solve the coupled ordinary differential Eqs. (11-13) together with the associated transformed boundary conditions in Eq.14 is the standard Newton-Raphson shooting method alongside the fourth-order Runge-Kutta integration algorithm. Let $f=x_{1}, f^{\prime}=x_{2}, f^{\prime \prime}=x_{3}, \theta=x_{4}, \theta^{\prime}=x_{5}, \phi=x_{6}, \phi^{\prime}=x_{7}$,

Equation 15 transforms Eq. 11 - Eq.13 to first order differential equations as follows:

$$
f^{\prime}=x_{1}^{\prime}=x_{2}, \quad f^{\prime \prime}=x_{2}^{\prime}=x_{3},
$$

$f^{\prime \prime \prime}=x_{3}^{\prime}=-x_{1} x_{3}+x_{2}^{2}-G_{T} x_{4}-G c x_{6}+(M+K)\left(x_{2}-1\right)-1$,

$$
\begin{gathered}
\theta^{\prime}=x_{4}^{\prime}=x_{5}, \\
\theta^{\prime \prime}=x_{5}^{\prime}=-1 /(1+4 / 3 R a)\left(\operatorname{Pr} x_{1} x_{5}+B r x_{3}^{2}+S x_{6}\right), \\
\phi^{\prime}=x_{6}^{\prime}=x_{7}, \\
\phi^{\prime \prime}=x_{7}^{\prime}=-S c x_{1} x_{7}+S c \beta x_{6}{ }^{n},
\end{gathered}
$$

subject to the boundary conditions

$$
x_{1}(0)=0, x_{2}(0)=0, x_{3}(0)=s_{1}, x_{4}(0)=1, x_{5}(0)=s_{2}, x_{6}(0)=1 \text {, }
$$$$
x_{7}(0)=s_{3}, x_{2}(\infty)=1, x_{4}(\infty)=0, x_{6}(\infty)=0 \text {. }
$$

In the shooting method, the unspecified initial conditions; $s 1, s_{2}$ and $s_{3}$ in Eq.17 were assumed and Eq.16 integrated numerically as an initial valued problem to a given terminal point. The accuracy of the assumed missing initial conditions is checked by comparing the calculated value of the dependent variable at the terminal point with its given value there. If differences exist, improved values of the missing initial conditions are obtained and the process repeated. The computations were done by a written programme which uses a symbolic and computational computer language (MAPLE). A step size of $\Delta \eta=0.001$ was selected to be satisfactory for a convergence criterion of $10^{-7}$ in nearly all cases. The maximum value of $\eta_{\infty}$ to each group of parameters are determined when the values of unknown boundary conditions at $\eta_{\infty}=0$ not change to successful loop with error less than $10^{-7}$. From the process of numerical computations, the local skin-friction coefficient, the local Nusselt numbers and the local Sherwood numbers, which are respectively, proportional to $f^{\prime \prime}(0),-\theta^{\prime}(0)$ and $-\phi^{\prime}(0)$ were worked out and their numerical values presented in tables.

Table 1 Comparison of results for different values of $S$ for $P r=G_{T}=1, G_{C}=S c=0.5, M=R a=B r=n=K=\beta=0$

\begin{tabular}{|l|l|l|l|l|l|l|l|l|l|}
\hline \multirow{3}{*}{$\boldsymbol{S}$} & \multicolumn{3}{|c}{ Singh et al. (2010) } & \multicolumn{3}{c|}{ Makinde (2011) } & \multicolumn{3}{c|}{ Present Study } \\
\cline { 2 - 11 } & $-\boldsymbol{f}^{\prime \prime}(\mathbf{0})$ & $-\theta^{\prime}(0)$ & $-\phi^{\prime}(0)$ & $-\boldsymbol{f}^{\prime \prime}(\mathbf{0})$ & $-\theta^{\prime}(0)$ & $-\phi^{\prime}(0)$ & $-\boldsymbol{f}^{\prime \prime}(\mathbf{0})$ & $-\theta^{\prime}(0)$ & $-\phi^{\prime}(0)$ \\
\hline-1 & $\mathbf{1 . 8 4 4 4}$ & 1.3908 & 0.4631 & $\mathbf{1 . 8 4 4 4 6 2}$ & 1.390856 & 0.463174 & $\mathbf{1 . 8 4 4 4 4 0 4}$ & 1.390859 & 0.463124 \\
\hline 0 & $\mathbf{1 . 9 9 9 5}$ & 0.6392 & 0.4789 & $\mathbf{1 . 9 9 9 5 5 3}$ & 0.639244 & 0.478964 & $\mathbf{1 . 9 9 9 5 5 4}$ & 0.639244 & 0.478965 \\
\hline 1 & $\mathbf{2 . 1 3 4 2}$ & -0.0730 & 0.4917 & $\mathbf{2 . 1 3 4 2 8 7}$ & -0.073040 & 0.491749 & $\mathbf{2 . 1 3 4 2 8 8}$ & 0.073041 & 0.491750 \\
\hline
\end{tabular}

Table 2 Numerical results of skin friction coefficient, Nusselt number and the Sherwood number

\begin{tabular}{|l|l|l|l|l|l|l|l|l|l|l|l|l|l|}
\hline$M$ & $P r$ & $S c$ & $G T$ & $G C$ & $B r$ & $\beta$ & $N$ & $S$ & $R a$ & $K$ & $f^{\prime \prime}(0)$ & $-\theta^{\prime}(0)$ & $-\phi^{\prime}(0)$ \\
\hline $\mathbf{1 . 0}$ & 0.71 & 0.22 & 0.1 & 0.1 & 0.1 & 0.1 & 1 & 1 & 0.1 & 1 & 1.976587 & -0.472184 & 0.354002 \\
\hline $\mathbf{3 . 0}$ & 0.71 & 0.22 & 0.1 & 0.1 & 0.1 & 0.1 & 1 & 1 & 0.1 & 1 & 2.429832 & -0.467904 & 0.359742 \\
\hline 0.1 & $\mathbf{4 . 0 0}$ & 0.22 & 0.1 & 0.1 & 0.1 & 0.1 & 1 & 1 & 0.1 & 1 & 1.712370 & 0.3037111 & 0.348490 \\
\hline 0.1 & $\mathbf{7 . 1 0}$ & 0.22 & 0.1 & 0.1 & 0.1 & 0.1 & 1 & 1 & 0.1 & 1 & 1.705917 & 0.627297 & 0.348150 \\
\hline 0.1 & 0.71 & $\mathbf{0 . 7 8}$ & 0.1 & 0.1 & 0.1 & 0.1 & 1 & 1 & 0.1 & 1 & 1.718618 & -0.208314 & 0.603392 \\
\hline 0.1 & 0.71 & $\mathbf{2 . 6 2}$ & 0.1 & 0.1 & 0.1 & 0.1 & 1 & 1 & 0.1 & 1 & 1.704956 & -0.012482 & 0.993426 \\
\hline 0.1 & 0.71 & 0.22 & $\mathbf{0 . 5}$ & 0.1 & 0.1 & 0.1 & 1 & 1 & 0.1 & 1 & 1.987683 & -0.450053 & 0.360734 \\
\hline 0.1 & 0.71 & 0.22 & $\mathbf{1 . 0}$ & 0.1 & 0.1 & 0.1 & 1 & 1 & 0.1 & 1 & 2.284949 & -0.426247 & 0.371958 \\
\hline 0.1 & 0.71 & 0.22 & 0.1 & $\mathbf{0 . 5}$ & 0.1 & 0.1 & 1 & 1 & 0.1 & 1 & 1.937509 & 0.457168 & 0.357961 \\
\hline 0.1 & 0.71 & 0.22 & 0.1 & $\mathbf{1 . 5}$ & 0.1 & 0.1 & 1 & 1 & 0.1 & 1 & 2.424761 & -0.420861 & 0.375171 \\
\hline 0.1 & 0.71 & 0.22 & 0.1 & 0.1 & $\mathbf{0 . 5}$ & 0.1 & 1 & 1 & 0.1 & 1 & 1.737424 & -0.778013 & 0.350365 \\
\hline 0.1 & 0.71 & 0.22 & 0.1 & 0.1 & $\mathbf{1 . 5}$ & 0.1 & 1 & 1 & 0.1 & 1 & 1.744340 & -1.536194 & 0.350681 \\
\hline 0.1 & 0.71 & 0.22 & 0.1 & 0.1 & 0.1 & $\mathbf{0 . 5}$ & 1 & 1 & 0.1 & 1 & 1.730358 & -0.407299 & 0.446052 \\
\hline 0.1 & 0.71 & 0.22 & 0.1 & 0.1 & 0.1 & $\mathbf{1 . 5}$ & 1 & 1 & 0.1 & 1 & 1.723057 & -0.291312 & 0.634837 \\
\hline 0.1 & 0.71 & 0.22 & 0.1 & 0.1 & 0.1 & 0.1 & $\mathbf{3}$ & 1 & 0.1 & 1 & 1.735537 & -0.492612 & 0.337513 \\
\hline 0.1 & 0.71 & 0.22 & 0.1 & 0.1 & 0.1 & 0.1 & $\mathbf{5}$ & 1 & 0.1 & 1 & 1.735739 & -0.495805 & 0.333211 \\
\hline 0.1 & 0.71 & 0.22 & 0.1 & 0.1 & 0.1 & 0.1 & 1 & $\mathbf{0}$ & 0.1 & 1 & 1.715682 & 0.428580 & 0.348837 \\
\hline 0.1 & 0.71 & 0.22 & 0.1 & 0.1 & 0.1 & 0.1 & 1 & $\mathbf{0 . 5}$ & 0.1 & 1 & 1.725223 & -0.025583 & 0.349543 \\
\hline 0.1 & 0.71 & 0.22 & 0.1 & 0.1 & 0.1 & 0.1 & 1 & 1 & $\mathbf{0 . 5}$ & 1 & 1.732679 & -0.284345 & 0.350227 \\
\hline 0.1 & 0.71 & 0.22 & 0.1 & 0.1 & 0.1 & 0.1 & 1 & 1 & $\mathbf{1 . 5}$ & 1 & 1.730886 & -0.100096 & 0.350273 \\
\hline 0.1 & 0.71 & 0.22 & 0.1 & 0.1 & 0.1 & 0.1 & 1 & 1 & 0.1 & $\mathbf{3}$ & 2.237210 & -0.468874 & 0.357483 \\
\hline 0.1 & 0.71 & 0.22 & 0.1 & 0.1 & 0.1 & 0.1 & 1 & 1 & 0.1 & $\mathbf{5}$ & 2.646350 & -0.468041 & 0.362016 \\
\hline
\end{tabular}




\section{RESULTS AND DISCUSSION}

From the process of numerical computation, the local skin-friction coefficient, the local Nusselt number and the local Sherwood number, which are respectively proportional to $-f^{\prime \prime}(0),-\theta^{\prime}(0)$, and $-\phi^{\prime}(0)$ were computed and their numerical values presented in a tabular form. Table 1 shows the comparison of the works of Singh et al. (2010) and Makinde (2011) with the present study for internal heat generation parameter $(S)$.It is clear from the table that our present study is consistent with the previous published results. The results of varying parameter values on the local skin friction coefficient, the local Nusselt number and the local Sherwood number, are shown in Table 2. It is observed that the local skin friction increases with increasing values of $M, G_{T}, G_{C}, B r, n, S$ and $K$ and decreases with increasing values of $P r, S c, \beta$ and $R a$. This means that the combined effect of magnetic field, buoyancy forces, viscous dissipation, order of chemical reaction, internal heat generation and porous medium permeability is to increase the local skin friction at the surface of the plate. Similarly, the rate of heat transfer at the plate surface increases with an increase in parameter values of $M, G_{T}, B r, K, P r, S c$, $\beta$ and $R a$ and reduces with increasing values of $G_{C}, n$ and $S$. Moreover, it is observed that the rate of mass transfer increases with increasing values of $M, G_{T}, G_{C}, B r, S, K, S c, \beta$ and $R a$ and decreases with increasing values of the order of chemical reaction and Prandtl number.

\subsection{Effects of Parameter Variation on Velocity Profiles}

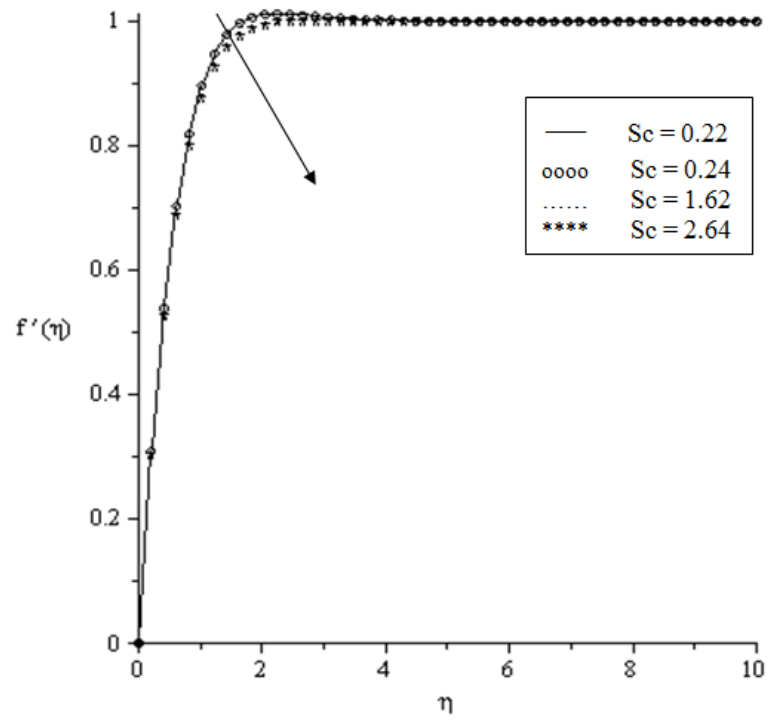

Fig. 2 Velocity profiles for varying values of Schmidt number

The effects of parameter variation on the velocity boundary layers are shown in Fig. 2-9. Normally, the fluid velocity is minimal at the plate surface and increases to the free stream value satisfying the far field boundary conditions. From Fig. 2 and Fig.7, it is observed that increasing values of the Schmidt and Prandtl numbers respectively tend to reduce the velocity profile. This can be attributed to the fact that increasing these two parameters sets momentum diffusion high over heat and mass diffusion at the surface of the plate which makes viscous force dominate the flow and hence, reduces the velocity at the boundary layer. The reaction rate parameter is also observed from Fig. 3 to reduce the velocity profile for obvious reasons. Moreover, it is observed from Fig. 6 that a steady decrease in the longitudinal velocity within the boundary layer accompanies a rise in the magnetic field intensity parameter with all profiles tending asymptotically to the free stream value away from the plate. This means in practice that, the magnetic field tends to decelerate the fluid flow. Meanwhile, in Fig. 8, it is observed that increasing the values of the internal generation parameter increases the velocity at the boundary layer. This can be attributed to the fact that, increase in the internal heat generation increases the fluid temperature which causes the fluid velocity to increase due to buoyancy effect. This fact is also adequate to explain the observed increase in the velocity profile as a result of increasing the thermal and solutal Grashof numbers in Fig. 4 and Fig. 9 respectively. We can note here that, increasing buoyancy forces will lead to a better flow kinematics. Moreover increasing the porous medium permeability increases the velocity at the surface of the plate as observed in Fig. 5.

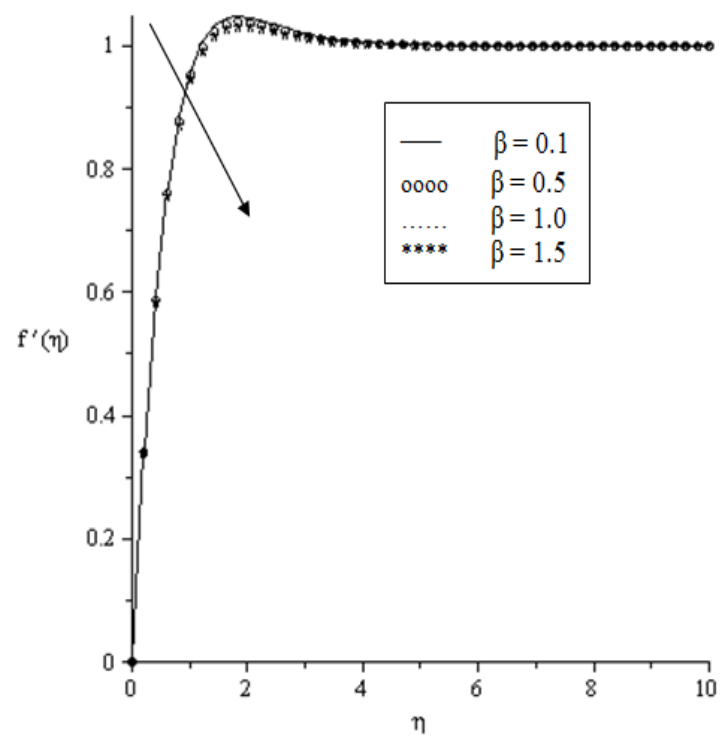

Fig. 3Velocity profiles for varying values of reaction rate parameter

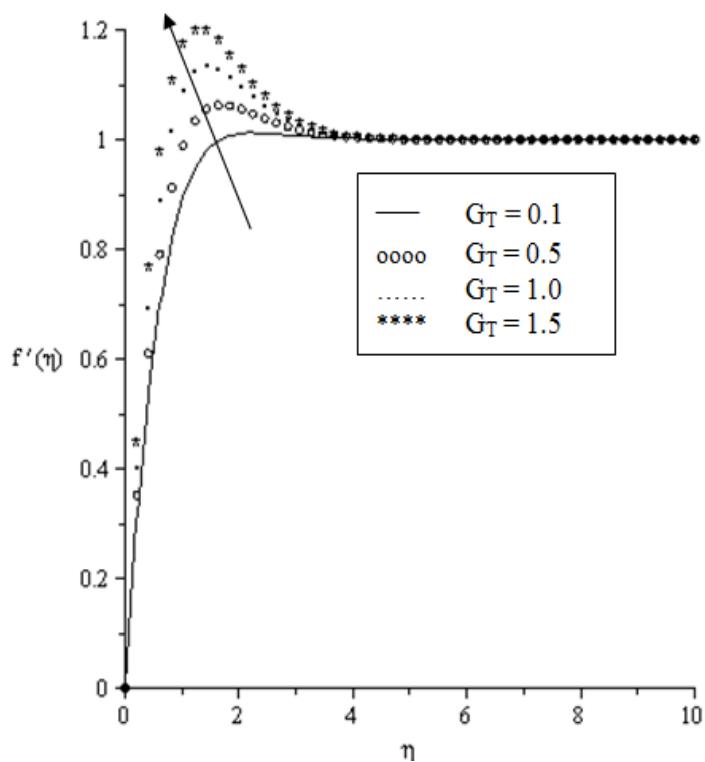

Fig. 4Velocity profiles for varying values of thermal Grashof number 


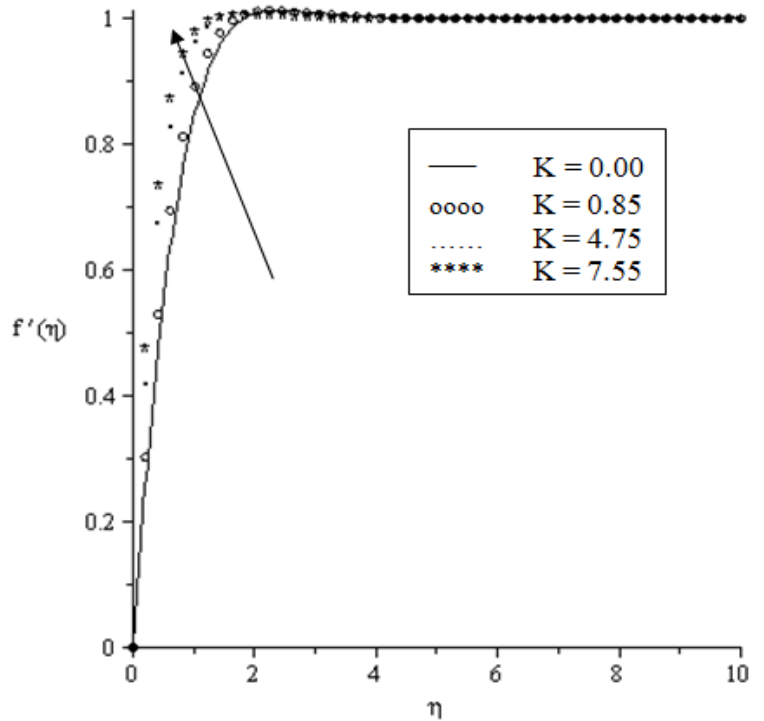

Fig. 5Velocity profiles for varying values of the permeability parameter

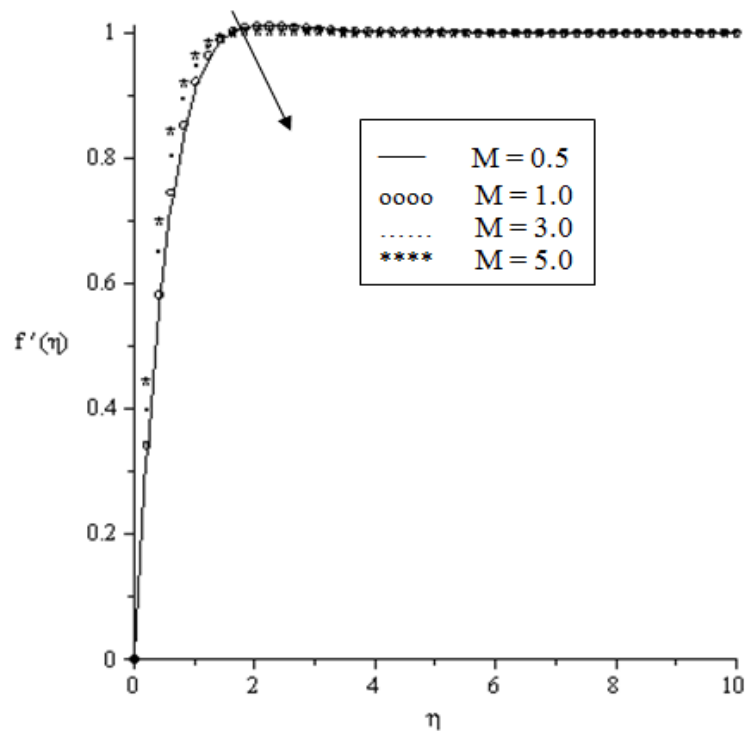

Fig. 6Velocity profiles for varying values of magnetic field parameter

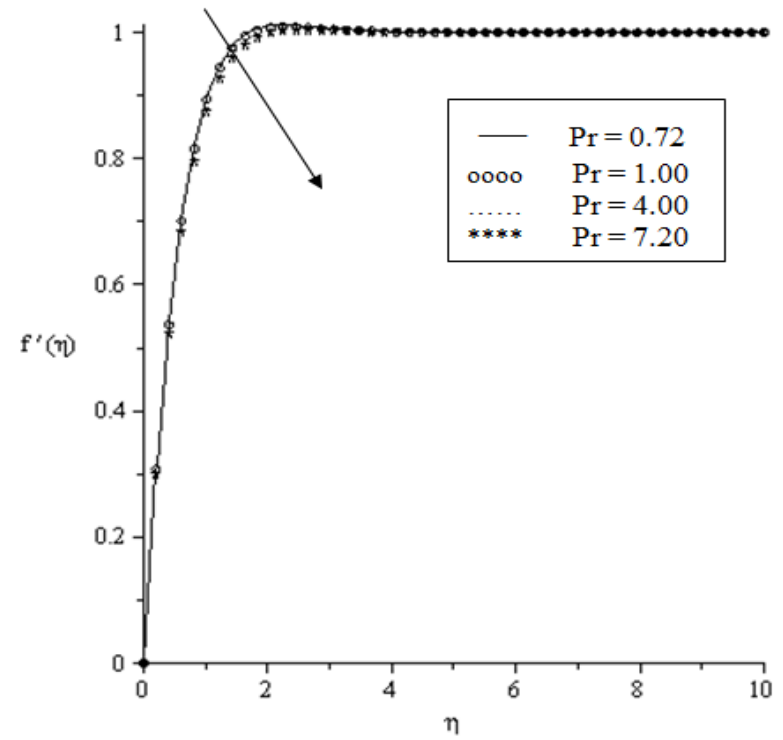

Fig. 7Velocity profiles for varying values of Prandtl number

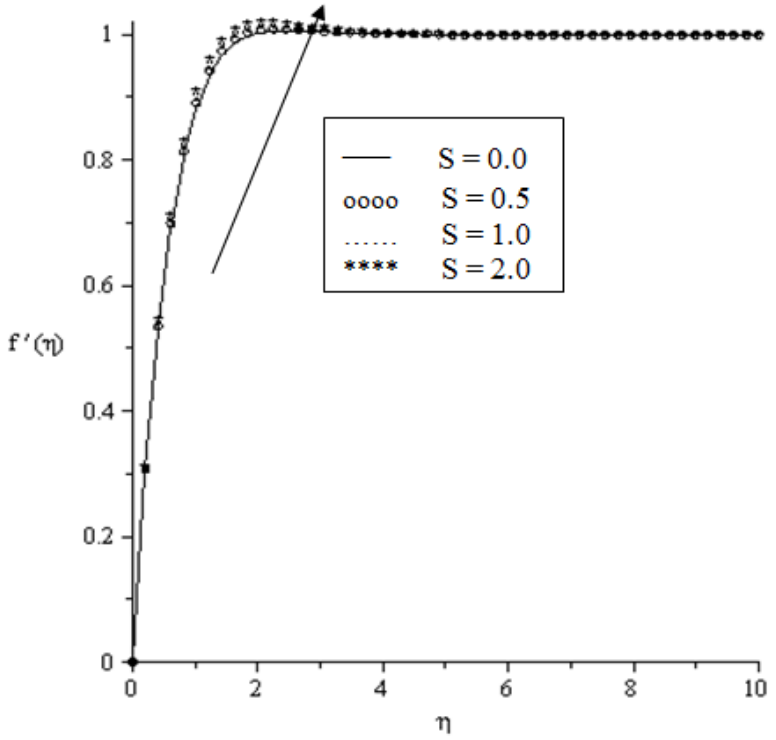

Fig. 8Velocity profiles for varying values of internal heat generation

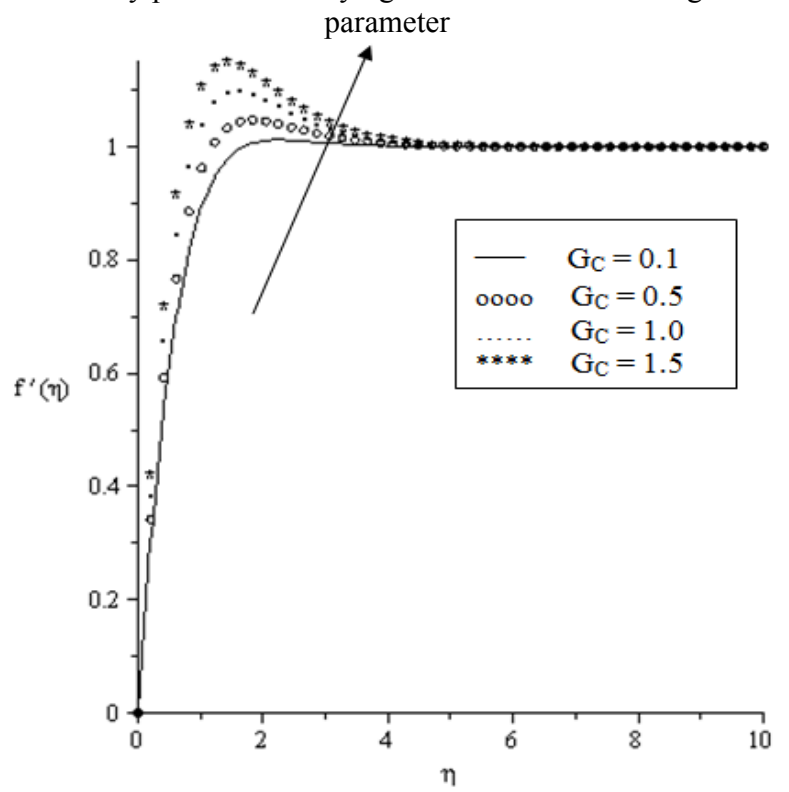

Fig. 9Velocity profiles for varying values of solute Grashof number

\subsection{Effects of Parameter Variation on Temperature Profiles}

The effects of parameter variation on temperature profiles are shown in Fig. 10-20. Normally, the fluid temperature is highest at the surface of the plate and shrinks to free stream temperature satisfying the boundary conditions. It is observed in Fig. 10, Fig. 18 and Fig. 19 that increasing viscous dissipation, thermal radiation and internal heat generation respectively increase the temperature profiles. This is due to the fact that as more heat is generated within the fluid, the fluid temperature increases leading to a sharp inclination of the temperature gradient between the plate surface and the fluid. Also, increasing the order of chemical reaction increases the temperature boundary layer thickness at the surface of the plate as observed in Fig. 16. Meanwhile, from Fig. 10, Fig. 14, Fig. 15, Fig. 17 and Fig. 20, it is observed that increasing the values of reaction rate parameter, porous medium permeability, magnetic field intensity, Prandtl number and Schmidt number respectively, reduce the thermal boundary layer thickness. Similarly, from Fig. 12 and Fig. 13, the temperature profiles are reduced as a result of increasing the buoyancy forces ( $G_{C}$ and $G_{T}$ respectively). This means that increasing the buoyancy forces enhance the cooling process. 


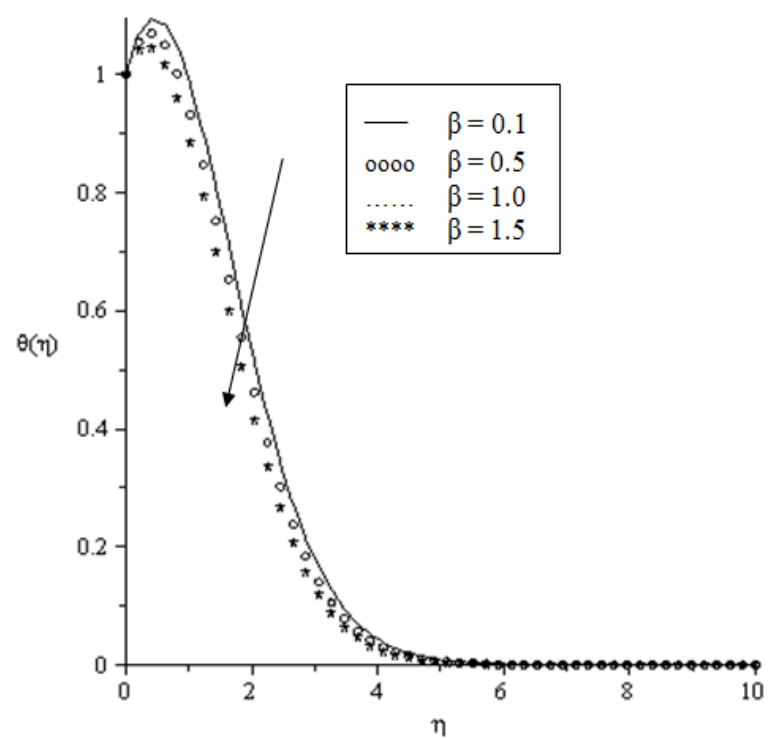

Fig. 10Temperature profiles for varying values of reaction rate

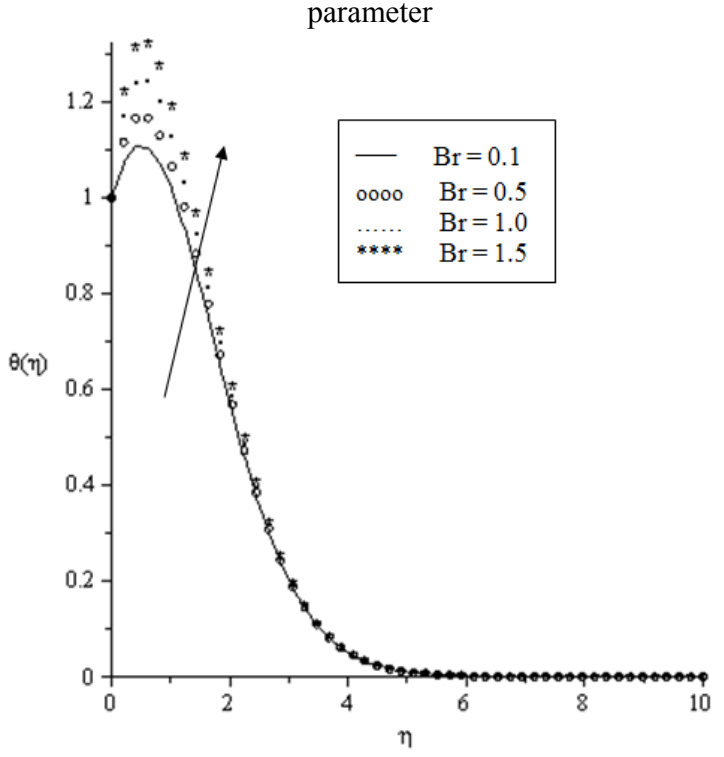

Fig. 11Temperature profiles for varying values of the Brinkman

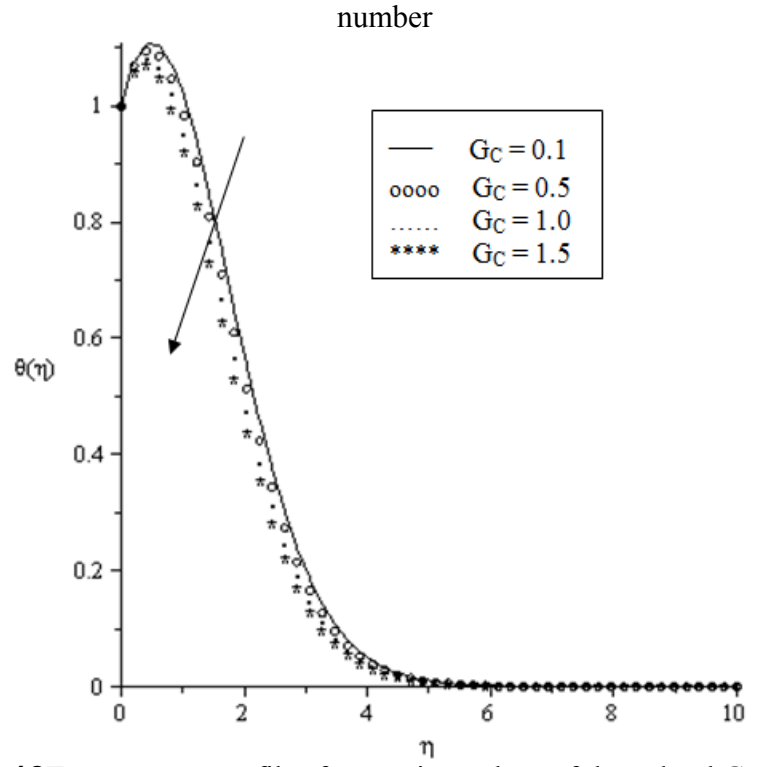

Fig. 12Temperature profiles for varying values of the solutal Grashof number

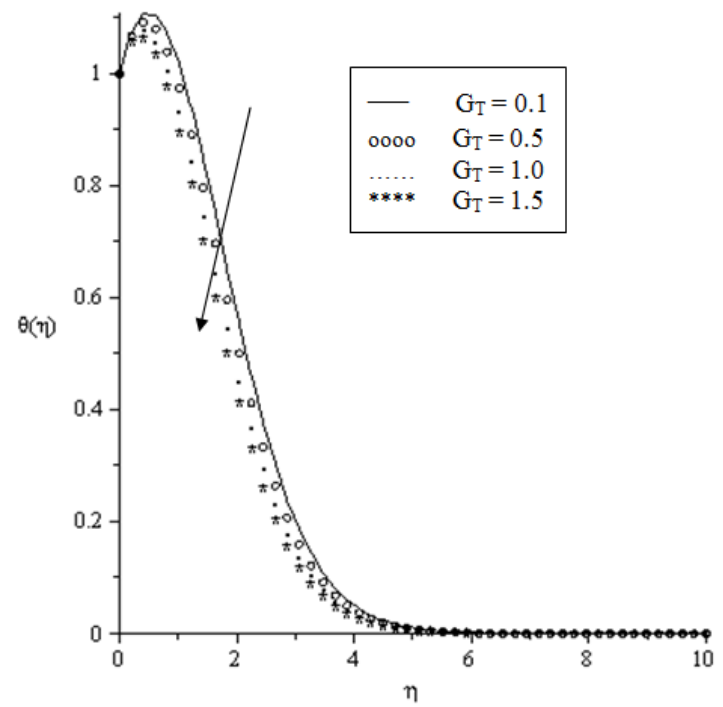

Fig. 13Temperature profiles for varying values of thermal Grashof number

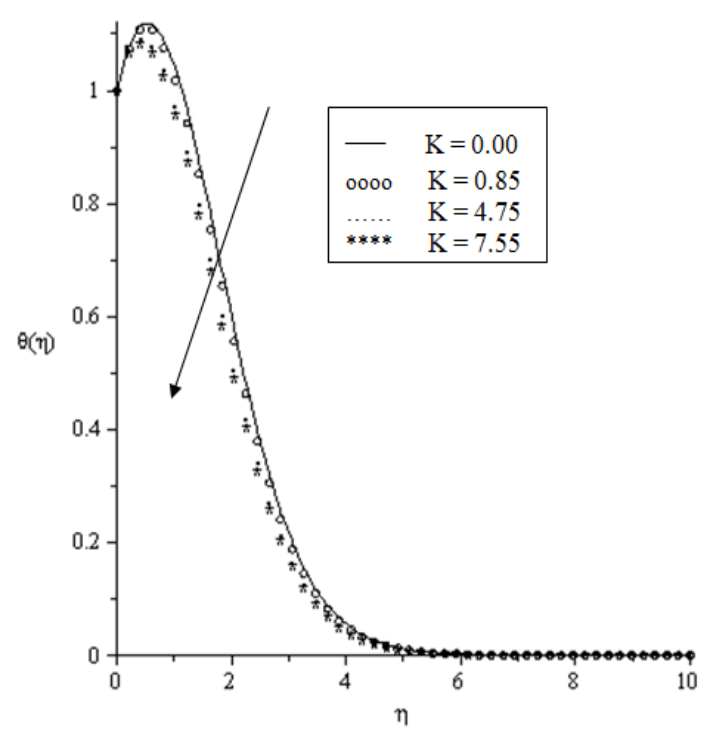

Fig. 14Temperature profiles for varying values of the permeability parameter

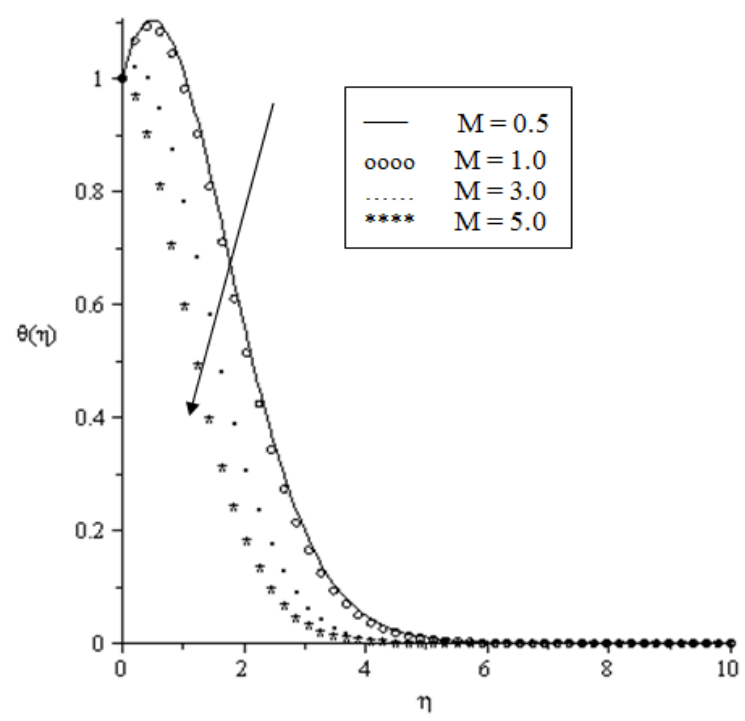

Fig. 15Temperature profiles for varying values of magnetic field parameter 


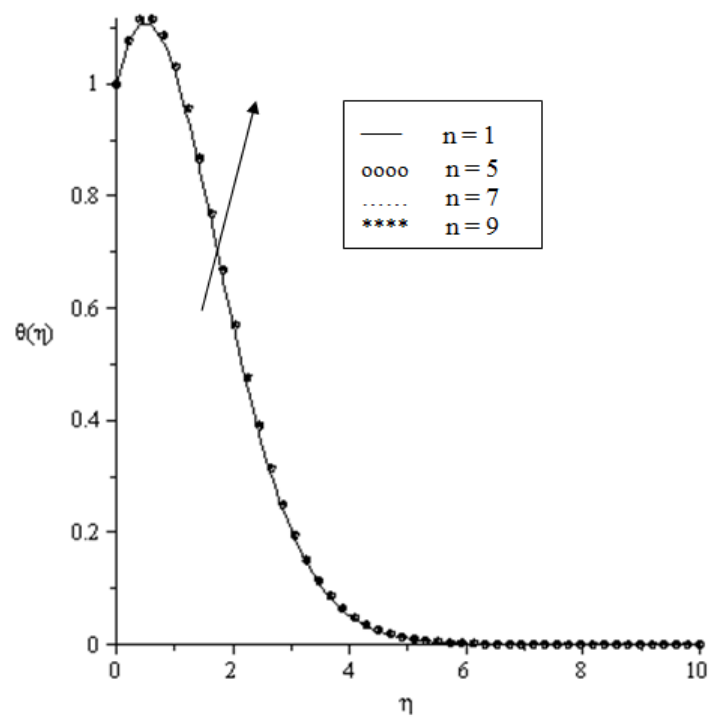

Fig. 16Temperature profiles for varying values of order of chemical reaction

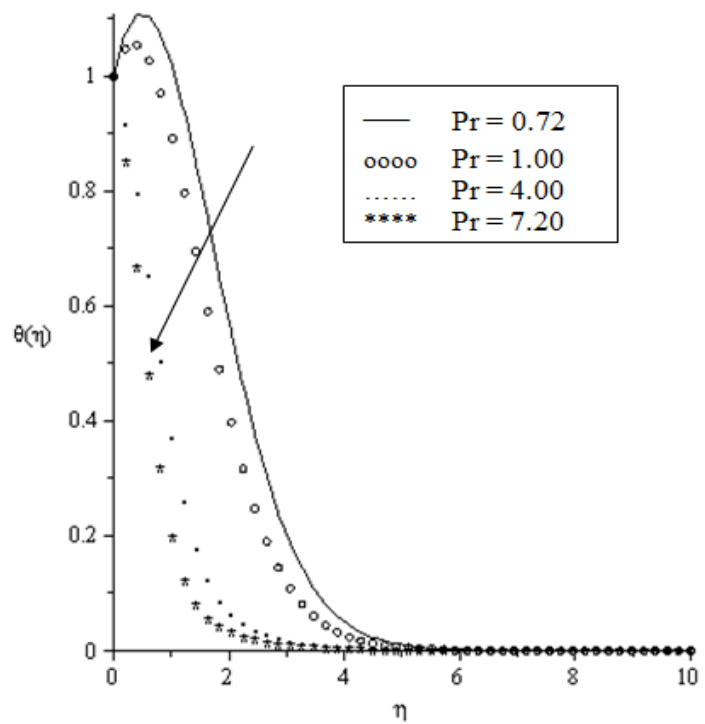

Fig. 17Temperature profiles for varying values of the Prandtl number

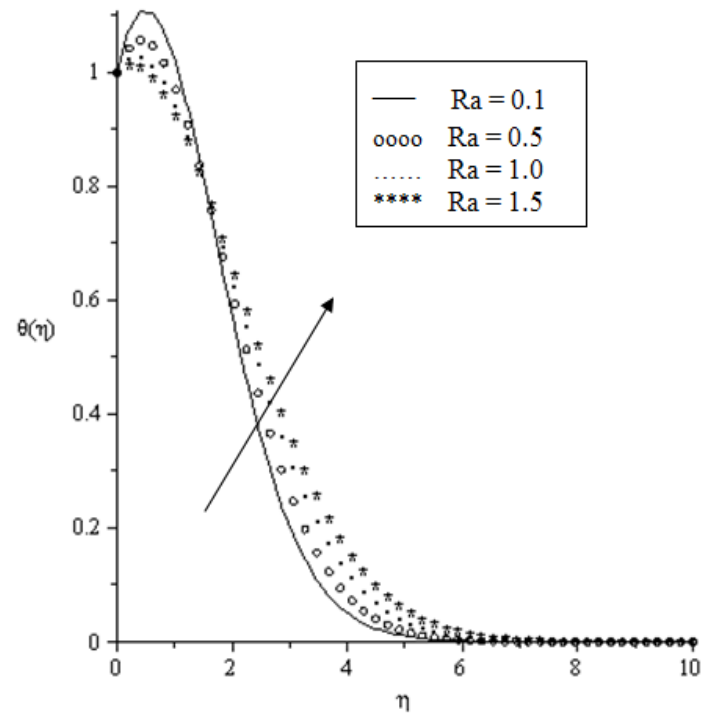

Fig. 18Temperature profiles for varying values of the radiation parameter

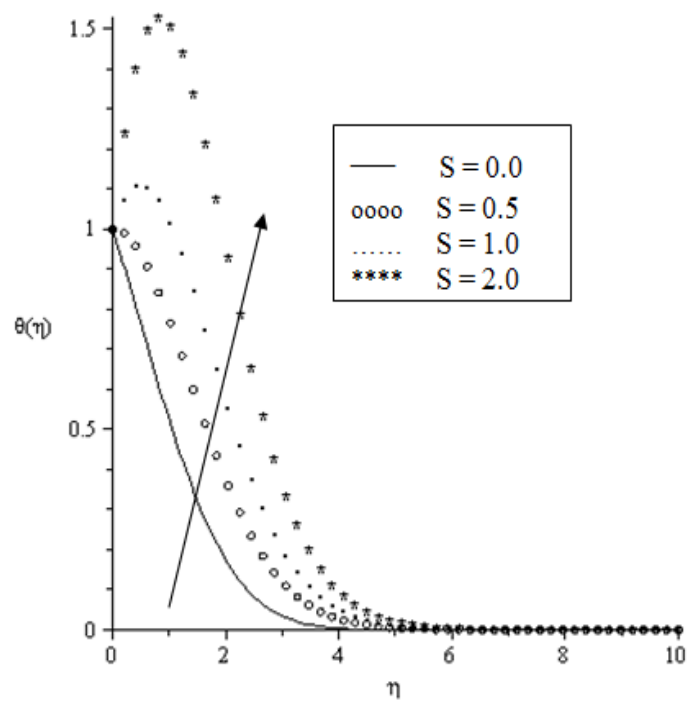

Fig. 19Temperature profiles for varying values of the internal heat generation parameter

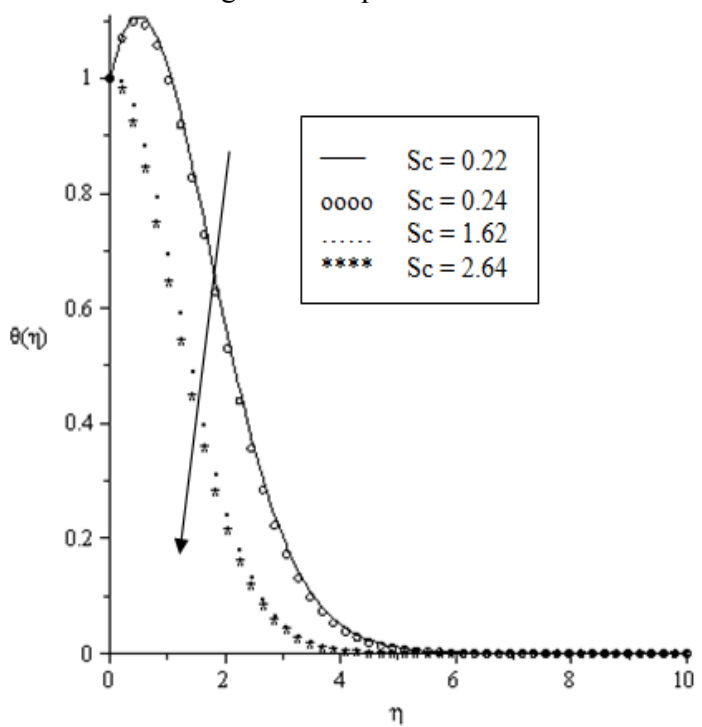

Fig. 20Temperature profiles for varying values of the Schmidt number

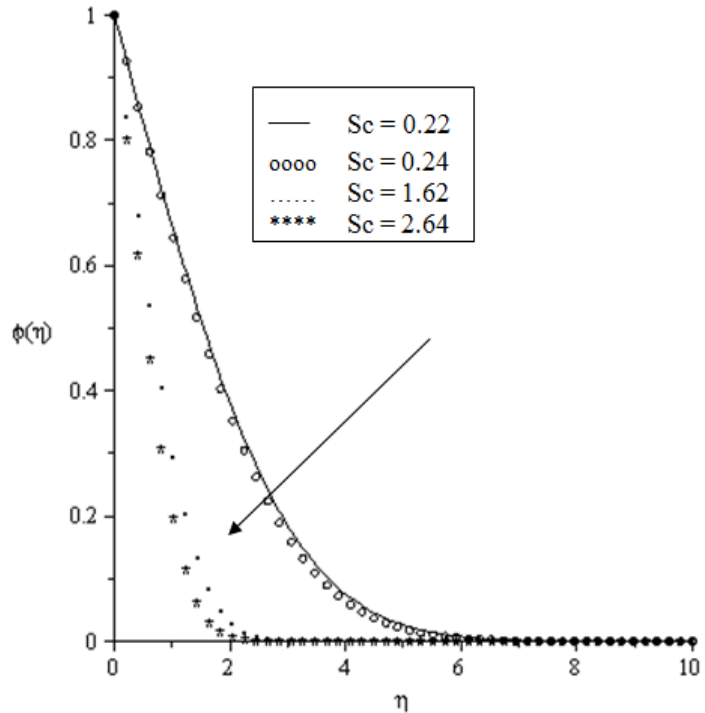

Fig. 21Concentration profiles for varying values of Schmidt number 


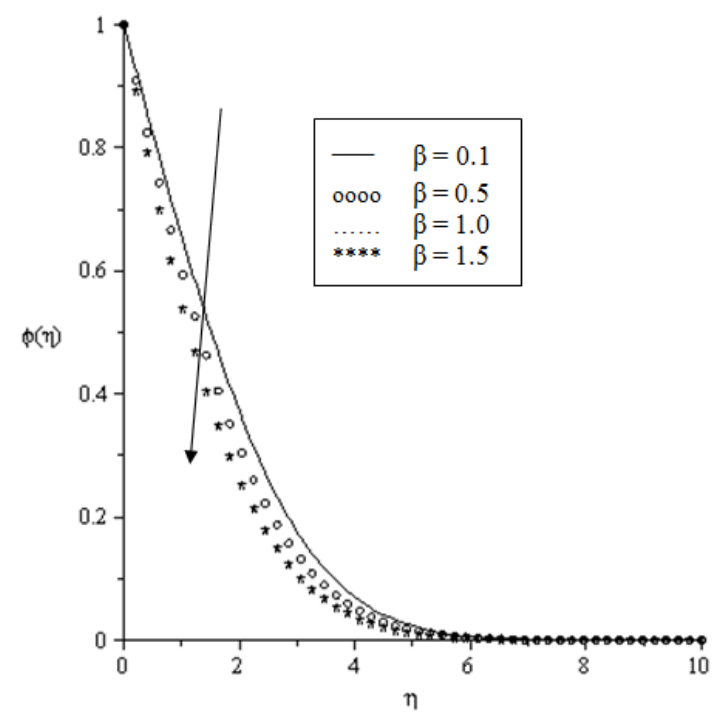

Fig. 22 Concentration profiles for varying values of reaction rate

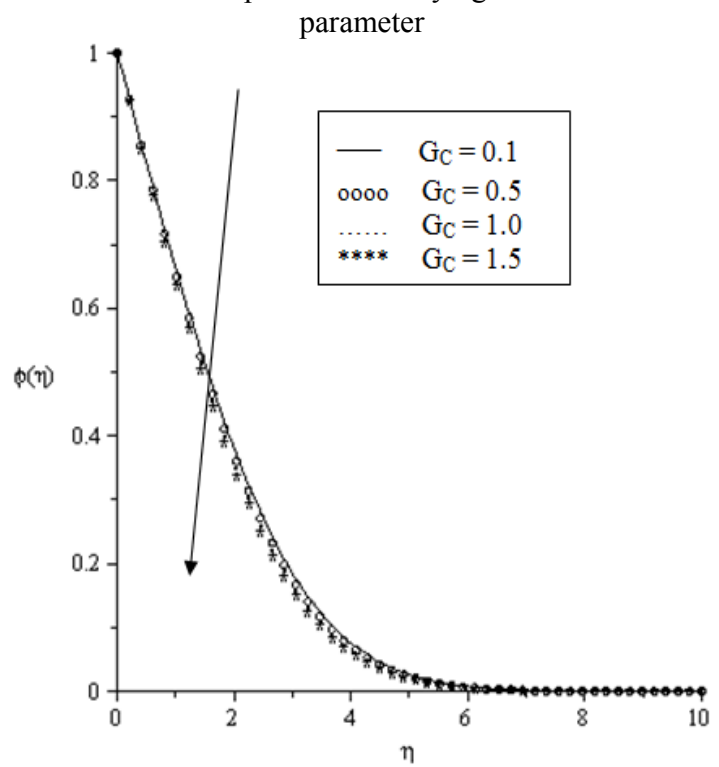

Fig. 23 Concentration profiles for varying values of solutal Grashof number

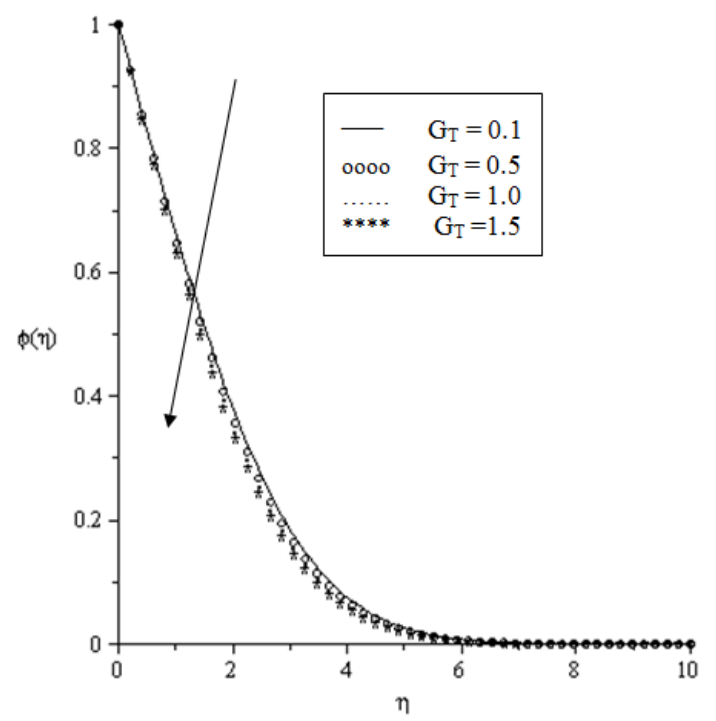

Fig. 24 Concentration profiles for varying values of thermal Grashof number

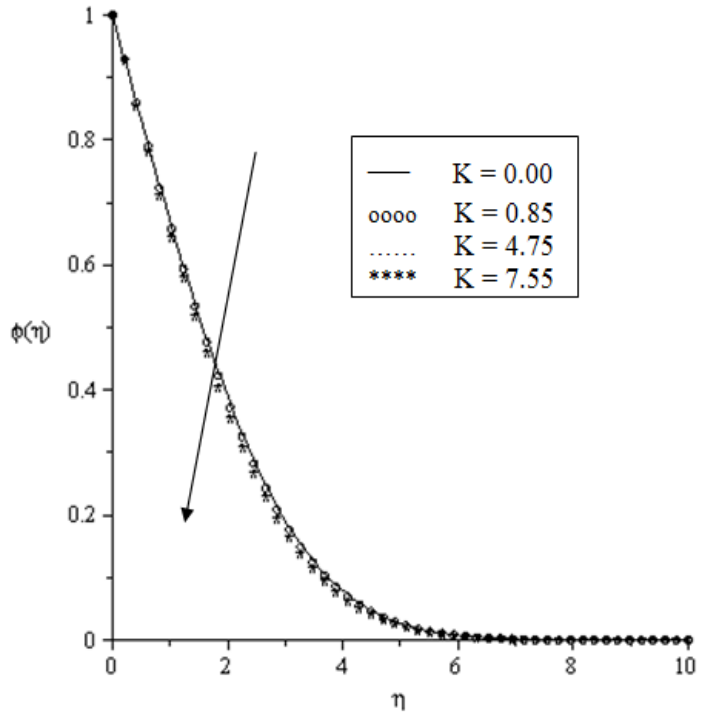

Fig. 25Concentration profiles for varying values of the permeability parameter

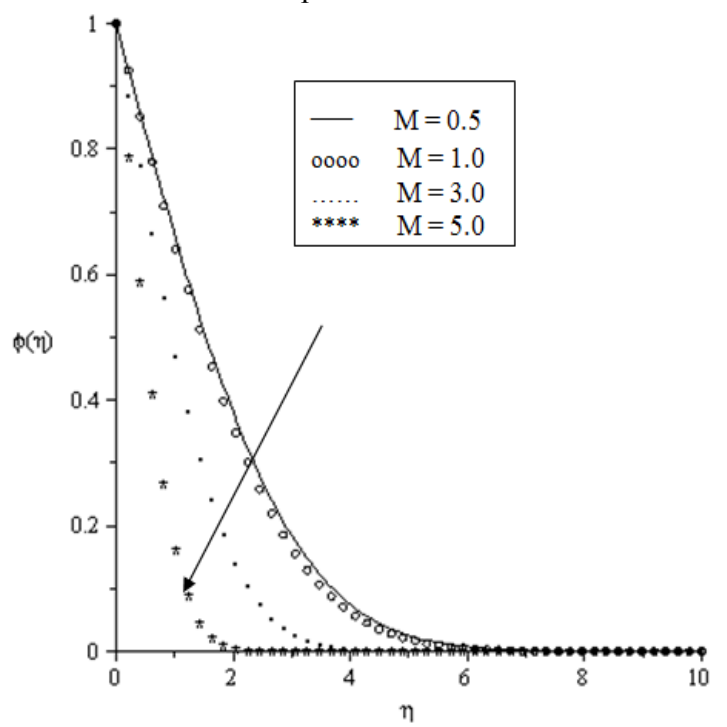

Fig. 26Concentration profiles for varying values of magnetic field parameter

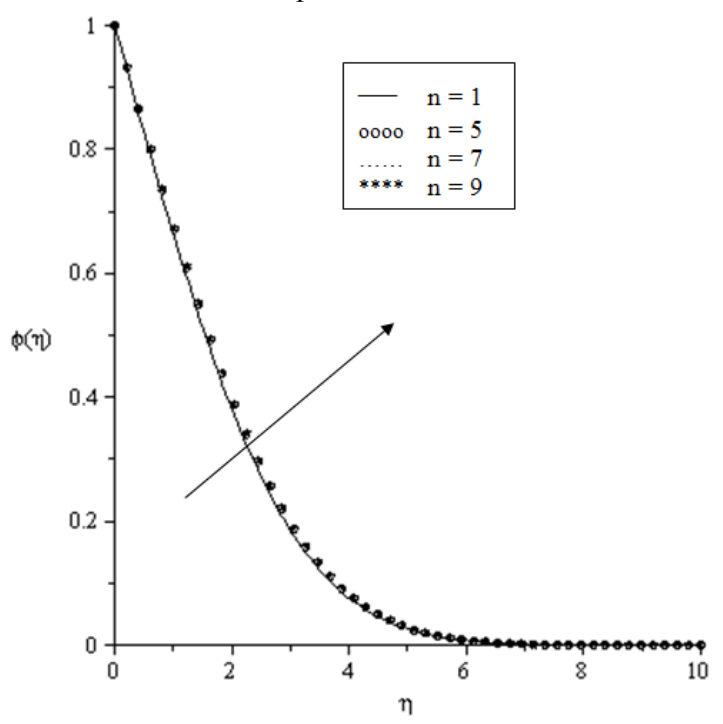

Fig. 27Concentration profiles for varying values of the order of chemical reaction 


\subsection{Effects of Parameter Variation on Concentration Profiles}

Figures 21-27 depict the effects of varying parameters on the concentration boundary layer thickness. Normally, the chemical species concentration is highest at the plate surface and reduces exponentially to the free stream zero value. It is observed that increasing the Schmidt number (Fig. 21), reaction rate (Fig. 22) buoyancy forces (Fig. 23 and Fig. 24), magnetic, porous medium permeability (Fig. 25) and field intensity (Fig. 26) have adverse effect of decaying the concentration boundary layer thickness. Meanwhile, the boundary layer thickness increases when the order of reaction increases as observed in Fig. 27.

\section{CONCLUSION}

Hydromagnetic fluid flow towards a vertical plate embedded in a saturated porous medium with thermal radiation, viscous dissipation, internal heat generation and $\mathrm{n}^{\text {th }}$ order chemical reactionhas been studied. Numerical results have been compared to earlier results published in the literature and a perfect agreement was achieved. Among others, our results reveal that:

i. The velocity increases with the increase in $M, S c, G_{T}, G_{C}, B r$ and $K$. It also decreases with the increase in $\operatorname{Pr}, \beta$, and $S$

ii. The temperature reduces with increasing $M, \operatorname{Pr}, S c, G_{T}, G_{C}, \beta$ and $K$; and also rises with increasing $R a, B r, n$ and $S$.

iii. The concentration boundary layer decreases with increasing $M, S c, G_{T}, G_{C}, \beta$ and $K$; and increases with increasing $n$.

iv. The skin friction at the surface increases for the increase in $M$, $G_{T}, G_{C}, B r, n, S$, and $K$; and decreases for increasing $P r, S c, R a$ and $\beta$.

v. The rate of heat transfer at the surface increases with the increase of $M, P r, S c, G T, B r, \beta, K$ and $R a$; and decreases with increasing $G_{C}, n$ and $S$.

vi. The rate of mass transfer at the surface increases with increasing $M, S c, G_{T}, G_{C}, B r, \beta, S, K$ and $R a$; and decreases with increasing $\operatorname{Pr}$ and $n$.

\section{ACKNOWLEDGEMENTS}

You may acknowledge the funding source of your work as well as suggestions and comments from, and discussions with colleagues in this section.

\section{NOMENCLATURE}

$(x, y) \quad$ Cartesian coordinates

$C_{w} \quad$ Plate surface concentration

$C_{\infty} \quad$ Free stream concentration

C Fluid chemical species concentration

D Diffusion coefficient

$f \quad$ Dimensionless stream function

$\mathrm{Nu} \quad$ Nusselt number

$T_{\infty} \quad$ Free stream temperature

Sh Sherwood number

$T \quad$ Fluid temperature

Pr Prandtl number

$T_{w} \quad$ Plate surface temperature

$(u, v) \quad$ Velocity components in Cartesian Coordinates

$\mathrm{K}^{\prime} \quad$ Mean absorption coefficient

$\check{K} \quad$ Permeability of the porous medium

$B_{0} \quad$ Magnetic field of constant strength

$q_{r} \quad$ Radiative heat flux

$\sigma^{*} \quad$ Stefan-Boltzmann constant

Greek Symbols

$\mu \quad$ Coefficient of viscosity
Density of the fluid

Dimensionless concentration

Dimensionless temperature

Dimensionless variable

Kinematic viscosity

Stream function

Fluid electrical conductivity

Thermal conductivity coefficient

Reaction rate

\section{REFERENCES}

Ali, F., Nazar, R., Arifin, N., and Pop, I., 2011, "Effect of Hall Current on MHD Mixed Convection Boundary Layer Flow over a Stretched Vertical Flat Plate," Meccanica, 46(5), 1103-1112

Arthur, E. M., and Seini, I. Y., 2014b, "Hydromagnetic Stagnation Point Flow over a Porous Stretching Surface in the Presence of Radiation and Viscous Dissipation," Applied and Computational Mathematics, 3(5), 191-196.

http://dx.doi.org/10.11648/j.acm.20140305.11

Arthur, E. M., Seini, I. Y., and Seidu, A., 2014, “'On Chemically Reacting Hydromagnetic Flow over a Flat Surface in the Presence of Radiation with Viscous Dissipation and Convective Boundary Conditions," American Journal of Applied Mathematics, 2(5), 179-185. http://dx.doi.org/10.11648/j.ajam.20140205.15

Arthur, E. M., and Seini, Y. I., 2014a, "MHD Thermal Stagnation Point Flow Towards a Stretching Porous Surface," Mathematical Theory and Modeling, 4(5), 163-169.

Bestman, A. R., and Adiepong, S. K., 1988, "Unsteady Hydromagnetic Free-Convection Flow with Radiative Heat Transfer in a Rotating Fluid," Astrophys Space Sci, 143, 73-80.

Chamkha, A. J., and Quadri, M. M. A., 2001, "Heat and Mass Transfer from a Permeable Cylinder in a Porous Medium with Magnetic Field and Heat Generation/Absorption Effects," Numerical Heat Transfer, Part A, Appl., 40(4), 387-401.

http://dx.doi.org/10.1080/104077801753238167

Chamkha, A. J., Issa, C., and Khanfer, K., 2002, "Natural Convection from an Inclined Plate Embedded in a Variable Porosity Porous Medium Due to Solar Radiation," Int. J. Therm. Sci., 41, 73-81. http://dx.doi.org/10.1016/S1290-0729(01)01305-9

Christian, E. J, Seini, Y. I, and Arthur, E. M., 2014, "MHD Boundary Layer Stagnation Point Flow with Radiation and Chemical Reaction towards a Heated Shrinking Porous Surface," International Journal of Physical Sciences, 9(14), 320-328.

http://dx.doi.org/10.5897/IJPS2014.4177

Gangadhar, K., 2012, "Similarity Solution for Natural Convection over a Moving Vertical Plate with Internal Heat Generation and Viscous Dissipation," Int. J. of Appl. Math and Mech., 8 (18), 90-100.

Hayat, T., Hameed, M. I., Asghar, S. S., and Iddiqui, A. M., 2004, "Some steady MHD flows of the second order fluid," Meccanica, 39, 345-355. http://dx.doi.org/10.1023/B:MECC.0000029365.39535.28

Ibrahim, M. S., and Bhashar, R. N., 2013, "Similarity Solution of Heat and Mass Transfer for Natural Convection over a Moving Vertical Plate with Internal Heat Generation and a Convective Boundary Condition in the Presence of Thermal Radiation, Viscous Dissipation, and Chemical Reaction," Hindawi Publishing Corporation ISRN Thermodynamics, 2013, Article ID 790604, 10 pages.

Ibrahim, S. Y., and Makinde, O. D., 2011, "Radiation Effect on Chemically Reacting MHD Boundary Layer Flow of Heat and Mass 
Transfer through a Porous Vertical Plate," International Journal of Physical Sciences, 6(6), 1508-1516.

Imoro, R., Arthur, E. M., and Seini, Y. I., 2014, "Heat and Mass Transfer over a Vertical Surface with Convective Boundary Conditions in the Presence of Viscous Dissipation and nth Order Chemical Reaction," International Journal of Computational and Applied Mathematics, 9(2), pp. 101-118, ISSN 1819-4966.

Ishak, A., Nazar, R., and Pop, I., 2006, "Mixed Convection Boundary Layers in the Stagnation-Point Flow toward a Stretching Surface," Meccanica, 41:509-518.

http://dx.doi.org/10.1007/s11012-006-0009-4

Ishak, A., Nazar, R., and Pop, I., 2008, "Mixed Convection Stagnation Point Flow of a Micropolar Fluid towards a Stretching Sheet," Meccanica, 43, 4110-418.

http://dx.doi.org/10.1007/s11012-007-9103-5

Kazi, K. H., Alim, M.d. A., and Laek, A. S., 2013, "Effects of Viscous Dissipation on MHD Natural Convection Flow along a Vertical Wavy Surface," Journal of Theoretical and Applied Physics, 2013, 7-31. http://www.jtaphys.com/content/7/1/31

Kumari, M., Takhar, H. S., and Nath, G., 2001, "Mixed Convection Flow over a Vertical Wedge Embedded in a Highly Porous Medium," Heat Mass Transf, 37, 139- 146.

http://dx.doi.org/10.1007/s002310000154

Magyari, E., and Pantokratoras, A., 2003, "Effect of Radiation in an Optically Thin Gray Gas Flowing Past a Vertical Infinite Plate in the Presence of a Magnetic Field," heat mass transf, 39, 771-773.

Makinde O. D., 2011, "Heat and Mass Transfer by MHD Mixed Convection Stagnation Point Flow toward a Vertical Plate Embedded in a Highly Porous Medium with Radiation and Internal Heat Generation," Meccanica, 2012(47), 1173-1184, http://dx.doi.org/10.1007/s11012-011-9502-5

Makinde, O. D., and Ogulu, A., 2008, "The Effect of Thermal Radiation on the Heat and Mass Transfer Flow of a Variable Viscosity Fluid Past a Vertical Porous Plate Permeated by a Transverse Magnetic Field," Chem. Eng Community, 195(12), 1575-1584.

http://dx.doi.org/10.1080/00986440802115549

Na, T. Y., 1979, "Computational methods in engineering boundary value problems," Academic Press, New York.
Naroua, H., Ram, P. C., Sambo, A. S., and Takhar, H. S., 1998, "FiniteElement Analysis of Natural Convection Flow in a Rotating Fluid with Radiative Heat Transfer," J. Magnetohydrodyn plasma Res, 7, 257-274.

Neild, D. A., and Bejan, A., 2006, "Convection in porous media," 3rd edn., Springer, New York.

Ouaf, M. E. M., 2005, "Exact Solution of Thermal Radiation on MHD, Flow over a Stretching Porous Sheet," Appl Math Comput, 170(2), 11171125 .

http://dx.doi.org/10.1016/j.amc.2005.01.010

Pantokratoras, A., 2004, "Effect of Viscous Dissipation in Natural Convection along a Heated Vertical Plate," Applied Mathematical Modelling, 29 (2005), 553-564.

http://dx.doi.org/10.1016/j.apm.2004.10.007

Raptis, A., Perdikis, C., and Leontitsis, A., 2003, "Effects of Radiation in an Optically Thin Gray Gas Flowing Past a Vertical Infinite Plate in the Presence of a Magnetic Field," Heat Mass Transf, 39, 771-773. http://dx.doi.org/10.1007/s00231-002-0317-8

Seddeek, M. A., 2002, "Effects of Magnetic Field and Variable Viscosity on Forced Non-Darcy Flow about a Flat Plate with Variable Wall Temperature in Porous Media in the Presence of Suction and Blowing," J. Appl Mech Tech Phys, 43, 13-17. http://dx.doi.org/10.1023/A:1013941523513

Singh, G., Sharma, P. R., and Chamkha, A. J., 2010, "Effect of Volumetric Heat Generation/Absorption on Mixed Convection Stagnation Point Flow on An Iso-Thermal Vertical Plate in Porous Media," Int. Math, 2(2), 59-71.

Singh, P. K., 2012, "Effects of Variable Fluid Properties and Viscous Dissipation on Mixed Convection Fluid Flow past a Vertical Plate in Porous Medium," International Journal of Scientific \& Engineering Research, 3(7), 2229-5518.

Sparrow, E. M., and Cess, R. D., 1978, "Radiation Heat Transfer, Hemisphere," Washington, Augmented edition.

Stuart, J. T., 1959, "The Viscous Flow near Stagnation Point when the External Flow has Uniform Vorticity,” Aerosp Sci Technol, 26, 124-125. http://dx.doi.org/10.2514/8.7963

Tamada, K., 1979, “Two-Dimensional Stagnation Point Flow Impinging Obliquely on a Plane Wall," J Phys Soc Jpn, 46, 310-311.

http://dx.doi.org/10.1143/JPSJ.46.310 